\title{
Interventional ultrasound beyond the classical abdominal applications. Are guidelines for other applications urgent needed?
}

\section{Zeno Sparchez}

\begin{abstract}
"Iuliu Hatieganu” University of Medicine and Pharmacy, Department of Gastroenterology, Regional Institute of
\end{abstract} Gastroenterology and Hepatology, Cluj-Napoca, Romania.

Interventional ultrasound (INVUS) has gained wide acceptance in the field of interventional radiology over the last years. The following advantages made US a very attractive guidance method in several applications: easy to use, possibility to perform and assess outcome and complications in real time, lack of radiation, repeatability, the possibility to perform at the bed side [1].

Abdominal ultrasound is a very popular imaging method being adopted by several medical and surgical specialties: internal medicine, gastroenterology, urology, nephrology, and oncology. Many applications both with diagnostic and therapeutic aims have been discovered and implemented in clinical practice over the last years [2]. For example in the last 10 years the number of papers with INVUS topics indexed in PubMed has dramatically increased (10652 vs.5011 before 2005).

Facing such a high number of interventions and clinical applications there are some concerns that have risen regarding its proper use, the knowledge of complication, availability and training.

Based on huge amount of knowledge and experience a group of physicians from Europe under the auspicion of European Society of Ultrasound in Medicine in Biology (EFSUMB) have recently elaborated Guidelines for INVUS. The guidelines will include recommendations regarding the general aspects of INVUS, diagnostic and therapeutic procedures using the transcutaneous and endoscopic ultrasound approaches and vascular access.

Received Accepted

Med Ultrason

2015, Vol. 17, No 3, 271-272

Corresponding author: Zeno Sparchez MD, PhD

Regional Institute for Gastroenterology and Hepatology

19-21Croitorilor str, Cluj Napoca, Romania

Email: zsparchez@yahoo.co.uk
However, the above-mentioned recommendations are addressed mainly to abdominal applications (using both the transabdominal and endoscopic ultrasound approach).

Interventional ultrasound has extensively widening its applications over the last years in many other fields (thyroid and other head and neck lesions like salivary glands tumors, soft tissue tumors, thorax lesions, musculoskeletal -MSK interventions, breast, obstetrics and gynecology, cardiology, anesthesiology, or intensive care medicine) [3-10]. A part of these interventions are performed by the interventional US physicians, mainly those with internal medicine specialty or by interventional radiologists (IRs) trained to perform INVUS [11].

Some of the non-abdominal applications of INVUS may be considered specialty-oriented interventions like MSK interventions in rheumatology and sports medicine, diagnostic and therapeutic procedures in obstetrics\&gynecology, cardiology and specific interventions in anesthesiology\&intensiv care medicine [6,7,911]. Regarding education, the training in INVUS in those fields should be part of a more complex US formation (usually with 3 levels of expertise) and may be included in the residency/fellowship programs. Experts from these fields should elaborate their own guidelines. However, the experience gained by other physicians, especially IRs is extremely helpful in the training process and also for guidelines publications.

In other fields (like thorax lesions, thyroid lesions and head\&neck tumors) although "interventional" US physicians and IRs perform many interventions there is an increasing number of procedures done by pulmonary medicine physicians, endocrinologists and otolaryngologists or head and neck surgeons $[5,13]$. In pulmonary medicine US training including INVUS has started to be part of residency programs in few countries for some years 
now but the process is slow. Possible explanations for this slow incorporation of US in pulmonary medicine are the paucity of training facilities and lack of knowledge and skills crossover with other specialties [5].

Finally there are some INVUS applications with less clear belonging to a speciality like breast and small parts tumors where radiologists do most of the interventions $[4,8]$. Practice may differ from country to country and even among different hospitals.

In these last 2 fields elaboration of guidelines are mandatory and should be done in an interdisciplinary way combining the big experience of "interventional" US physicians and/or IRs with the clinical experience of physicians with those specialties. For those fields but also for the first group of applications EFSUMB, as a multidisciplinary society, may provide the support to elaborate and publish guidelines.

\section{References}

1. Dodd GD 3rd1, Esola CC, Memel DS, et al. Sonography: the undiscovered jewel of interventional radiology. Radiographics 1996 ; 16: 1271-1288.

2. Dietrich CF, Nuernberg D. Interventional ultrasound. Stuttgart: Thieme Publisher, 2014.

3. Blank W, Braun B. Sonography of the thyroid-part 2: thyroid inflammation, impairmant of thyroid function and interventions. Ultraschall Med 2008; 29: 128-149.
4. Freuis T, Loizides A, Gruber H. Ultrasound guided core needle biopsy of soft tissue tumors; a fool proof technique? Med Ultrason 2011; 13: 187-194.

5. Stigt JA, Groen HJ. Percutaneous ultrasonography as imaging modality and sampling guide for pulmonologists. Respiration. 2014; 87: 441-451

6. Finnoff JT, Hall MM, Adams E, et al. American Medical Society for Sports Medicine position statement: interventional musculoskeletal ultrasound in sports medicine. Clin J Sport Med 2015; 25: 6-22.

7. D'Agostino MA, Schmidt WA. Ultrasound-guided injections in rheumatology: actual knowledge on efficacy and procedures. Best Pract Res Clin Rheumatol 2013; 27: 283-294.

8. Plantade R. Interventional radiology: the corner-stone of breast management. Diagn Interv Imaging. 2013; 94: 575-591.

9. D. Lemery , J. Santolaya-Forgas . Interventional Ultrasound in Obstetrics, Gynaecology and the Breast., WileyBlackwell, 1998

10. Silvestry FE, Kerber RE, Brook MM et al. Echocardiography-guided interventions. J Am Soc Echocardiogr 2009; 22: 213-231.

11. Grau T, Mäcken T, Strunk H. Appendix 13: Intensive care ultrasound - minimum training requirements for the practice of medical ultrasound in Europe. Ultraschall Med 2009; 30: 414-417.

12. The Royal College of Radiologist. Ultrasound training recommentadions for medical and surgical specialities. Second edition. London. The Royal College of Radiologists, 2012.

13. Pfeiffer J, Ridder GJ. Diagnostic value of ultrasound-guided core needle biopsy in patients with salivary gland masses. Int J Oral Maxillofac Surg 2012; 41: 437-443. 